\title{
Monitoring the on-orbit calibration of Terra MODIS reflective solar bands using simultaneous Terra MISR observations
}

\author{
Amit Angal ${ }^{\mathrm{a}}$, Xiaoxiong (Jack) Xiong ${ }^{\mathrm{b}}$, and Aisheng $\mathrm{Wu}^{\mathrm{a}}$ \\ ${ }^{\mathrm{a} S}$ Science Systems and Applications Inc. 10210 Greenbelt Road, Lanham, MD 20706 \\ ${ }^{\mathrm{b}}$ Sciences and Exploration Directorate, NASA Goddard Space Flight Center, Greenbelt, MD
}

\begin{abstract}
On December 18, 2015 Terra spacecraft completed 16 years of successful operation in space. Terra has five instruments designed to facilitate scientific measurements of the Earth's land, ocean, and atmosphere. The Moderate Resolution Imaging Spectroradiometer (MODIS) and the Multi-Angle Imaging Spectroradiometer (MISR) instruments provide information for the temporal studies of the globe. After providing over 16 years of complementary measurements, a synergistic use of the measurements obtained from these sensors is beneficial for various science products. The 20 reflective solar bands (RSB) of MODIS are calibrated using a combination of solar diffuser and lunar measurements, supplemented by vicarious measurements from pseudo-invariant desert sites. MODIS views the on-board calibrators and the Earth via a two-sided scan mirror at three spatial resolutions: $250 \mathrm{~m}$ using 40 detectors in bands 1 and 2, $500 \mathrm{~m}$ using 20 detectors in bands 3 and 4, and 1000m using 10 detectors in bands 8-19 and 26. Simultaneous measurements of the Earth's surface are acquired in a push-broom fashion by MISR at nine view angles spreading out in the forward and backward direction along the flight path. While the swath width for MISR acquisitions is $360 \mathrm{~km}$, MODIS scans a wider swath of $2330 \mathrm{~km}$ via its two-sided scan mirror. The reflectance of the MODIS scan mirror has an angle dependence characterized by the response versus scan-angle (RVS). Its on-orbit change is derived using the gain from a combination of on-board and Earth-view measurements. The on-orbit RVS for MODIS has experienced a significant change, especially for the short wavelength bands. The on-orbit RVS change for the short wavelength bands (band 8, 9, 3) at nadir is observed to be greater than $10 \%$ over the mission lifetime. Due to absence of a scanning mechanism, MISR can serve as an effective tool to evaluate and monitor the on-orbit performance of the MODIS RVS. Furthermore, it can also monitor the detector and scan-mirror differences for the MODIS bands using simultaneous measurements from Earth-scene targets, e.g. North Atlantic Ocean and North African desert. Simultaneous measurements provide the benefit of minimizing the impact of Earth-scene features while comparing the radiometric performance using vicarious techniques. Long-term observations of both instruments using select ground targets also provide an evaluation of the long-term calibration stability. The goal of this paper is to demonstrate the use of MISR to monitor and enhance the on-orbit calibration of the MODIS RSB. The radiometric calibration requirements for the MODIS RSB are $\pm 2 \%$ in reflectance and $\pm 5 \%$ in radiance at typical radiance levels within $\pm 45^{\circ}$ of nadir. Results show that the long-term changes in the MODIS reflectance at nadir-frames are generally within $1 \%$. While the impact due to changing polarization sensitivity is evident in some blue bands, the mirror side differences for other MODIS bands are observed to be within $1 \%$, therefore demonstrating an excellent on-orbit calibration performance. The detector differences in the blue bands of MODIS exhibit divergence in recent years beyond $1 \%$ and a calibration algorithm
\end{abstract}


improvement has been identified to mitigate this effect. Short-term variations in the recent year caused by the forward updates were identified in bands 1 and 2 and are planned to be corrected in the next reprocess.

\section{Introduction}

The instruments on the Terra mission continue to provide valuable scientific observations of the Earth's land, ocean and atmosphere after more than 16 years of on-orbit operation. MODIS (Moderate Resolution Imaging Spectroradiometer) is a key instrument aboard the Terra platform and its observations are used to generate a wide range of land, ocean, and atmospheric science products. A key performance parameter impacting the quality of the science data products is the calibration stability. In addition to an intensive prelaunch calibration and characterization, MODIS uses a set of onboard calibrators (OBCs) to regularly track the on-orbit calibration of the instrument [1], [2]. The reflective solar bands (RSB) of MODIS range from 0.41 to $2.1 \mu \mathrm{m}$, and are calibrated via regular solar diffuser (SD) and lunar observations, supplemented by the Earth scene observations from the pseudoinvariant desert targets. As the MODIS instrument continues to operate well beyond its design lifetime (6 years), an accurate characterization of the sensor behavior becomes extremely challenging. The Multi-Angle Imaging Spectroradiometer (MISR) instrument on the Terra spacecraft, also with a design lifetime of 6 years, continues to operate successfully providing several key land and atmosphere data products [3], [4], [5]. MODIS and MISR (nadir camera) observe the earth-scene targets simultaneously therefore providing a unique opportunity to have a synergistic use of the various data-products derived from these sensors.

In contrast to the $360 \mathrm{~km}$ swath width of MISR, MODIS has a wider swath of $2330 \mathrm{~km}$ achieved using a two-sided scan mirror with a $\pm 55^{\circ}$ scan range. With a push-broom imaging mechanism, MISR observations are centered on the MODIS swath (at nadir). The four spectral bands of MISR $(0.46,0.55,0.67$, and $0.86 \mu \mathrm{m})$ overlap with several MODIS land, atmosphere, and ocean color spectral bands. Previous work focused on assessing the calibration difference between these two sensors using widely accepted inter-comparison techniques. Radiometric validation of both MISR and MODIS was also performed via field campaigns over the Railroad Valley (RRV) playa in Nevada, USA [4]. Long-term top-of-atmosphere (TOA) reflectance trending over pseudo-invariant calibration sites (PICS), with standard corrections for bi-directional reflectance factor (BRDF), relative spectral response (RSR) differences etc. was used to evaluate the calibration differences between the two sensors [6], [7], [8]. Recently, an automated approach to ground-based vicarious calibration has been operational using the Radiometric Calibration Test Site (RadCaTS) located at the RRV site [9]. The observations from these efforts provide an understanding of the calibration mechanism used by these sensors along with the various uncertainties associated with it. The goal of this paper is to demonstrate the use of MISR to effectively monitor and enhance the on-orbit calibration of MODIS RSB in support of maintaining the calibration accuracy of the MODIS level 1B (L1B) products.

MODIS views the Earth's surface and its onboard calibrators using a two-sided scan mirror. In addition to a dependence on the wavelength of incident light, the reflectance of the scan mirror also depends on the angle of incidence (AOI). The dependence of the scan mirror's reflectance on the AOI is described by the response versus scan-angle (RVS) function characterized prelaunch and monitored on-orbit using the SD, lunar and Earth-scene 
observations. Although MISR does not cover the entire MODIS swath, it can be effectively used to validate the onorbit RVS performance of the MODIS RSB around nadir using near-simultaneous Earth-scene observations. Similarly the scan mirror reflectance differences can also be assessed using these simultaneous observations. In the case of the short-wavelength visible bands, the detector to detector calibration difference exhibits scan angle dependence in addition to being different for both mirror sides. Consequently, a detector-dependent RVS has been implemented for these bands in the MODIS collection 6 (C6) algorithms [10], [11], [12], [13]. The detector gain is derived using the solar diffuser observation collected at $50.25^{\circ}$ and the detector differences from the SD and on-orbit lunar measurements are used to derive the detector-dependent RVS. Residual detector differences caused by uncertainties in the on-orbit gain manifest in the form of detector-detector striping in the MODIS L1B products. Near-simultaneous MISR observations of Earth-scene targets are used to assess the performance and identify enhancements for the short-wavelength bands. Finally, the long-term calibration stability of MODIS RSB is also assessed using these near simultaneous MISR observations from two Earth-scene targets, the North African desert and North Atlantic Ocean, to accommodate the low-gain and high-gain MODIS RSB.

The paper is organized into the following sections: Section 2 discusses the details of MODIS instrument including its on-orbit calibration mechanism as well as an overview of MISR instrument; Section 3 discusses the methodology; and the results are included in Section 4. Finally, the summary and conclusions are in Section 5.

\section{Background and Motivation}

\section{a. MODIS RSB calibration and Collection 6 overview}

Since its launch in December 1999, the MODIS instrument on the Terra spacecraft has been acquiring Earth-scene and onboard measurements on a continuous basis. Given the longevity of the MODIS mission, an unprecedented amount of instrument measurements have been acquired and processed. The focus of this paper is to formulate a mechanism to independently validate and enhance the on-orbit calibration of MODIS RSB using MISR. Some key details regarding the Terra MODIS instrument and its operation, calibration, and on-orbit performance can be found here [13]. The 20 reflective solar bands on MODIS cover the spectral range from 0.41 to $2.1 \mu \mathrm{m}$. Bands 1 and 2, with nadir spatial resolutions of $250 \mathrm{~m}$, are designed for land applications. Bands 3-7, with nadir spatial resolutions of $500 \mathrm{~m}$ are designed for land and atmosphere applications, and Bands 8-19 and 26 with1 $\mathrm{km}$ nadir spatial resolutions are designed for ocean and atmospheric applications.

The MODIS RSB are calibrated at a detector level using regular SD observations with the degradation of the SD tracked using regular operations of the solar diffuser stability monitor (SDSM). As introduced earlier, the MODIS RSB calibration needs to account for the AOI-dependent reflectance change of the MODIS scan mirror. In the C5 algorithm (started from YYYY), this is achieved by coupling the response of the solar diffuser acquired at $50.25^{\circ}$

with the near-monthly lunar observations acquired at $11.25^{\circ}$ with a linear behavior assumed at all other angles. The scan mirror AOIs are reported in the L1B granule for each of the1354 frames in the scan line. The frame number F and the AOI are related by 


$$
\theta=\frac{65.5^{\circ}-10.5^{\circ}}{1353} F+10.5^{\circ}
$$

where $\mathrm{F}$ varies from 0 to 1353 . In this paper, the scan-angle dependence of MODIS RSB will be denoted as a function of frame.

The degradation of the MODIS SD as monitored by the SDSM has shown strong wavelength dependence. The degradation rates range from $50 \%$ at $412 \mathrm{~nm}$ to about $2 \%$ at $936 \mathrm{~nm}$. The degradation of the SD is accounted for in the computation of the instrument gain. As the instrument continues to operate beyond its design lifetime of 6 years, the degradation of the SD is inadequately estimated [12]. Also, the assumption of the linear change of response at frames between SD and lunar gain does not appear to hold. To compensate for this, Earth scene measurements from pseudo-invariant desert sites are used to track the long-term change at various scan-angles. This approach is currently implemented in C6 for Terra MODIS bands 1-4, 8-10 and will be extended to other bands as needed. Also, a detector-dependent RVS has been applied to bands 3, 8-12 and its extension to other RSB is also planned in the future.

Figures 1a-d show the prelaunch RVS and its on-orbit variation for the four representative Terra MODIS bands 9, 4, 1 and 2, respectively. The RVS has been plotted as a function of frames for three representative years, 2005, 2010 and 2015. The RVS, as implemented in the C6 L1B, is a fitted function; hence the trends presented in Figure 1 exhibit a smooth change over the frame range. The short wavelength band $9(443 \mathrm{~nm})$ shows a change of about $12 \%$ at the start of scan for the most recent year. The band $4(555 \mathrm{~nm})$ shows a change of about $5 \%$ for the most recent year, and the changes observed in bands 1 and 2 are less than 2\%. The changes at nadir for band 9 exceed 5\% whereas they are observed to be within $3 \%$ for the remaining bands. The large changes observed in the on-orbit RVS trends are a reflection of the instrument's behavior and are corrected for in the calibrated L1B product. An effective means to evaluate the accuracy of the RVS corrections is to measure the scan-angle dependence in the calibrated L1B product while looking at a uniform Earth-scene target. In order to avoid the various complexities associated with an Earth scene target, MISR can be used as an effective substitute to evaluate these changes.

\section{b. MISR instrument and its suitability for RSB calibration monitoring}

MISR acquires Earth observations via nine cameras pointed at fixed angles, with one pointing at nadir and four each viewing the forward and backward directions along the spacecraft ground track. The forward and aftward cameras are symmetrically arranged around nadir image at $26.1^{\circ}, 45.6^{\circ}, 60^{\circ}$, and $70.5^{\circ}$. MISR has a $3 \%$ absolute calibration requirement in radiance scale [4], [5]. Using measurements from its onboard calibrators, supplemented by vicarious calibration measurements, the MISR calibration is monitored and updated on-orbit. The onboard calibrators consist of two Spectralon diffuse panels and six sets of photo-diode detectors designed to measure the reflected solar irradiance from the panels. The response is then compared with each camera's digital output, thereby providing the on-orbit radiometric calibration for MISR [5]. 
This study is restricted to only the nadir-camera (AN) since it acquires images at a similar view-angle as MODIS. Also, it provides imagery that is less distorted by surface topographic effects in comparison with other cameras. Unlike the whiskbroom scanning mechanism of MODIS, MISR employs a push-broom mechanism while acquiring the Earth-scene images. Due to the absence of a scan mirror, co-located MODIS-MISR observations can be used to assess the MODIS scan mirror side differences. Although MISR has a narrower swath compared to MODIS, it can still be used to validate the RSB RVS performance over $\pm 10^{\circ}$ around nadir. Statistically significant co-located MODIS-MISR pixels can also facilitate an evaluation of any possible MODIS detector-biases. If the long-term calibration stability of MISR is assumed, it can also provide useful information regarding the MODIS radiometric calibration stability on-orbit.

Figure 2 shows the comparison between the RSRs of the two instruments, with MODIS plotted in black and MISR plotted in pink. The solid-black lines represent the RSR for the MODIS land bands and the dotted lines represent the RSR for the MODIS ocean bands. The RSR for the four MISR bands (blue, green, red, and near-infrared) is observed to have a wider bandwidth in comparison with the MODIS ocean bands and a narrower bandwidth in comparison with the MODIS land bands. Since the goal of this paper is to demonstrate the use of MISR to monitor and enhance the on-orbit characterization of MODIS RSB, a correction for RSR mismatch is not required.

\section{Methodology}

\section{a. Site-and data-selection (North Atlantic Ocean and North African desert)}

Since MODIS and MISR are acquiring Earth-scene measurements from the same platform with a similar viewangle, selection of a fixed target should not be required. However, selection of a fixed pseudo-invariant target on the Earth's surface can significantly reduce the various uncertainties associated with the surface and atmospheric properties. Given the diverse scope of this work (RVS validation, detector- and mirror-side difference, long-term stability) and different characteristics of MODIS bands (spectral and gain settings), a single ground target is insufficient. Hence, two different targets of varying characteristics are chosen for this study. The widely used North African desert, located at $28.6^{\circ} \mathrm{N}, 23.4^{\circ} \mathrm{E}$ is selected. In addition to providing year-round measurements for both instruments, the desert site also presents unsaturated measurements for some of the MODIS ocean bands. Also, a dark-ocean site is necessary to extend the comparison and analysis to the high-gain ocean bands of MODIS; the North Atlantic ocean site located around $24^{\circ} \mathrm{N}, 42^{\circ} \mathrm{W}$ is also selected. A fixed number of MISR blocks are selected from every overpass of these sites over the mission lifetime. Figure 3 shows the selected blocks from a MISR overpass of each site. The image presented is from the red- MISR bands and has been stretched and arranged appropriately for illustration purposes. A same number (6) of MISR blocks are selected from each overpass. The region represented by these blocks as shown below was consistently chosen across the entire mission lifetime for consistency.

The MODIS L1B Collection 6 granules were obtained from the LAADS (https://ladsweb.nascom.nasa.gov/). The MISR datasets were obtained from the NASA Langley Research Center Atmospheric Science Data Center. In addition to the TOA reflectance/radiance products, the corresponding geolocation files were also obtained. About 
350 desert and ocean overpasses were used in this analysis. In the case of MODIS, the analysis was confined to the 1 $\mathrm{km}$ aggregated products for ease of geo-location matchup with MISR. The MODIS-MISR scene-pairs from each of these sites occur on a repeating cycle of once every 16 days.

\section{b. Band-averaged and detector-dependent radiance/reflectance calculation}

The MODIS L1B granules cover a 5-minute interval with swath dimensions of $2330 \mathrm{~km}$ (cross track) by $10 \mathrm{~km}$ (along-track at nadir). Due to the narrower swath of MISR, a small area centered on nadir in the cross track direction can be selected for this analysis. As discussed earlier, the MISR blocks covering the MODIS granule are selected and used to obtain co-located MODIS and MISR pixels. In order to obtain the MODIS geo-located pixels at $1 \mathrm{~km}$ resolution, the MODIS L1A geolocation product (L1AGEO) is used to retrieve the latitude and longitude values for each pixel. The distance between a MODIS and a MISR-pixel is computed based on the retrieved latitude and longitude values using the following expression

$$
d=\sqrt{\left[X_{\text {MISR }}(i, j)-X_{\text {MODIS }}(k, l)\right]^{2}+\left[Y_{M I S R}(i, j)-Y_{M O D I S}(k, l)\right]^{2}}
$$

where $\mathrm{i}$ and $\mathrm{j}$ are the cross track and along track indices for MISR respectively, and $\mathrm{k}$ and $\mathrm{l}$ denote the cross track and along track indices for MODIS. A smaller value of $d$ corresponds to a larger overlap between the pixel pair and in order to retain pixels with a significant overlap a threshold of $0.0025^{\circ}$ (which corresponds to a distance of $250 \mathrm{~m}$ ) is used as a threshold. After this thresholding, at least 20,000 matched pixels are retained for each of the North Africa and the North Atlantic scene-pair. For each matched pixel between the two sensors, a ratio of top-ofatmosphere reflectance is computed using the following expression

$$
r=\rho_{\text {MODIS }}(b, m, f, d) / \rho_{\text {MISR }}(b)
$$

where the $\rho_{\text {MODIS }}$ and $\rho_{\text {MISR }}$ denote the TOA reflectance for MODIS and MISR and the indices $\mathrm{b}, \mathrm{m}, \mathrm{d}$, $\mathrm{f}$ denote the spectral band, mirror side, detector number and frame number. Only the spectrally matching bands as shown in Figure 2 are chosen for this analysis. Since MISR does not acquire the data using a scanning mechanism as MODIS, it can be an effective tool in assessing the reflectance differences between the two sides of the MODIS scan mirror. The pushbroom mechanism can also be used to evaluate the RVS performance of the MODIS RSB in the calibrated L1B product. The reflectance ratio can be plotted as a function of MODIS frames to assess the performance of the MODIS RVS. The reflectance ratio can also be used to assess any residual detector-detector differences in the MODIS L1B product. These evaluations can be performed due to the fact that MISR has exhibited a pixel relative calibration to within $0.5 \%$ [4]. Finally, the reflectance ratios can also be used to track the long-term radiometric stability of the MODIS spectral bands over the selected sites. This assessment is based on the underlying assumption that the on-orbit calibration of MISR complies with its radiometric calibration stability specification [4], [6]. 


\section{Results:}

\section{a. MODIS RVS monitoring (at frames corresponding to MISR swath)}

The MODIS RSB RVS was characterized prelaunch using the SIS-100 by illuminating the scan-mirror at 12 different frames at three lamp levels, corresponding to three radiance levels. The on-orbit variation of the RVS is tracked using the measurements from onboard calibrators (primarily SD and lunar) and supplemented by the response trends from pseudo-invariant desert targets. While the on-orbit RVS characterization for bands 1,2,4 and 9 uses the lunar and desert observations, the RVS for bands 12, 13,14 and 16 continues to rely on the onboard calibrators. The performance of the RVS can be evaluated by tracking the MODIS/MISR reflectance ratio as a function of the MODIS frame number. Figure4a shows the MODIS/MISR reflectance ratio plotted as a function of frame number for a simultaneous overpass of the North African desert from 2005. The result for all the detectors (mirror side 1) of band 9 and corresponding MISR blue band are shown. A linear model is applied to this data to estimate the deviation of the MODIS/MISR reflectance ratio between frames 500 and 800 . The deviation from unity is attributed to the RSR difference between the spectral bands. Although, MODIS is viewing the desert region for a majority of the scan, scene variations will have an impact on the observed reflectance as a function of frame. The Earth-scene features observed by MODIS across this frame range may also not be uniform and normalizing by MISR eliminates the dependence on ground features. Since the acquisitions are simultaneous and near-nadir, the effects of atmospheric and surface BRDF are assumed to be consistent across this small frame range. Figure 4b shows a similar result for band 12 from an ocean scene-pair acquired in 2005. The ocean granules are impacted by the sun-glint that results in a saturated response for the high-gain ocean bands. This effect is evident in Figure 4b from frame 700 onwards. Also, the deviation of the MODIS/MISR ratio along the fitted line is observed to vary by up to $10 \%$ as opposed to around $5-7 \%$ for the desert scene-pairs. Ideally, a single ground surface is desirable to evaluate the RVS performance for all of the MODIS bands considered here; however, the ocean bands (12, 13, 14 and 16) saturate while viewing the desert and the land bands yield a very low signal while viewing the ocean sites.

The relative difference between the MODIS/MISR ratio at frame 500 and frame 800 is computed for each scene-pair on a band and mirror side basis and is shown in equation (3), where b denotes the band, m denotes the mirror side and $t$ denotes the time of the overpass

$$
\text { relative }- \text { difference }(b, m, t)=\frac{\rho_{\text {MODIS }-f r 500}(b, m, t) / \rho_{M I S R-f r 500}(b, t)}{\rho_{\text {MODIS }-f r 800}(b, m, t) / \rho_{M I S R-f r 800}(b, t)}
$$

This ratio is computed by performing a simple linear fit to the ratios as a function of frame number. A value of this ratio around unity reaffirms the RSB on-orbit RVS characterization. A calendar-year average has been performed on the derived results to better highlight and understand the observed trends as shown in Figures $5 \mathrm{a}$ to $5 \mathrm{~d}$. The results for band 1 and 4, derived from the desert scene-pairs exhibit a stable temporal trend with changes between years 2000 and 2015 within 1\%. The results for MODIS bands 13 and 14, presented in Figures 5c and 5d, are derived from the ocean scene-pairs. Although the long-term trend is observed to be within 1\%, the observed standard deviations for the ocean-granules are significantly larger than the desert granules. A likely reason for this behavior is 
a combination of several scene-dependent phenomenon i.e. sun-glint, pixels contaminated by clouds etc. A complete summary for all the MODIS RSB chosen for this study is presented in Table I. The results from four representative years along with the standard deviation values are presented. In general, the results presented in Table I indicate the temporal change of the relative difference to be within $1 \%$ with greater standard deviation values for the oceanscene-pairs in comparison with the desert scene-pairs. The results presented in Table I, indicate that no significant bias exists between the response at frames 500 and 800, which further confirms the performance of the on-orbit RVS for MODIS RSB.

\section{b. MODIS mirror side differences}

The reflective properties between the two sides of the scan mirror are known to be different since the prelaunch characterization, with more divergence observed from on-orbit measurements. The magnitude of this response variation exhibits a wavelength as well as AOI dependence. Although the mirror-side dependent gain of the instrument at any AOI should account for this change, residual mirror side differences may still exist in the calibrated L1B product [14], [15]. One of the primary reasons behind this is the changing polarization property of the MODIS scan-mirror. The impact is largest at short wavelengths with a prominent dependence on scene type and view geometry [16]. Since MISR uses the same pushbroom detectors for adjacent observations, normalizing each mirror side by MISR effectively removes variations due to scene content. It should be noted that this approach would be most effective for the MODIS spectral bands which have the largest overlap with MISR.

By collating all the matched pixels from each mirror side, the difference in the TOA reflectance for both mirror sides can be estimated. Figure 6 shows the reflectance mirror side ratios for four representative MODIS RSB bands. In each case, the MODIS-reflectance for each mirror side has been normalized by MISR to eliminate the impact due

to Earth-scenes. The results for bands 4 and 9 are derived from the desert sites whereas the bands 13 and 16 results are derived from ocean granules. Although fluctuations of upto $\pm 1 \%$ are observed, the long-term change for all bands considered here is less than $2 \%$. In the case of band 9, deviation from unity is observed starting in 2009. This is likely caused by the change in the polarization property of the scan mirror, which is different in magnitude for each mirror side. The polarization effect is also wavelength dependent, with the largest impact observed at short wavelengths. It should be noted that the MODIS L1B does not involve a correction for any changes in the polarization sensitivity, but the down-stream MODIS products do incorporate a correction for this effect.

\section{c. MODIS detector-detector differences}

The MODIS $1 \mathrm{~km} \mathrm{L1B}$ products are used in the study to evaluate the detector-detector differences for the MODIS RSB after normalization using MISR reflectance. In the case of MODIS bands 1 and 2 (40 detectors and 4 subframes) and band 4 (20 detectors and 2 subframes), the $1 \mathrm{~km} \mathrm{L1B}$ product performs an aggregation so that information is retrieved at $1 \mathrm{~km}$ spatial resolution (at nadir). The ocean bands $(9,12,13,14$, and 16) considered in this study acquires data at $1 \mathrm{~km}$ spatial resolution at nadir via 10 detectors. Using the reflectance ratio from equation (2), the MODIS detector-detector differences for a given band, mirror side are evaluated. The measurements from the desert are used for the evaluation of bands 1, 2, 4 and 9, and the ocean data is used for bands 12, 13, 14 and 16. 
Figure 7 (a)-(d) shows the detector difference (i.e. MODIS/MISR ratio normalized to the mean) for bands 4, 9, 12 and 16. The results for mirror side 1 are presented and a calendar year average is performed to identify long-term deviations. The different colors in each plot represent different detectors and the error bars denote the standard deviation. The large standard deviation observed for bands 12 and 16 is expected as the trends are derived from the ocean granules that have significant impacts due to atmospheric scattering [17]. With the exception of band 9, the long term spread between the detectors is within $0.5 \%$ for all the bands. This is also true for the bands not presented in the Figure. In the case of band 9, the difference between detector 1 (black) and detector 10 (light blue) begins to evolve after 2010 and reaches a magnitude of about 1\% in 2015. As discussed earlier, a detector-dependent RVS is applied to the band 9, which is derived from the detector dependent lunar and SD gains. The lunar measurements are acquired at frame 17, and the SD gains are measured at frame 978. Consequently, a linear interpolation is used derive the detector differences at nadir. It is evident from the trends observed in band 9, that the linear approximation for the detector differences is inadequate and an improvement in the current approach is necessary.

\section{d. MODIS long-term trending}

The longevity of MODIS time-series measurements also warrants a necessity of highly accurate long-term radiometric calibration stability. Due to an inadequacy in the calibration approach, the MODIS L1B from Collection 5 (the predecessor to C6) exhibited long-term drifts up to 8\% in some of the short-wavelength bands of Terra MODIS. In order to overcome the shortcomings of C5, the improved algorithm involved supplementing the measurements of the on-orbit calibrators with Earth-view response trends from pseudo-invariant desert targets. The improved algorithm, as implemented in C6, has demonstrated significant improvement notably in the mitigation of the long-term drifts at short wavelengths. The MODIS Characterization Support Team (MCST) continues to monitor the radiometric stability via Earth-scene observations. In addition to providing an independent validation source for MODIS absolute calibration, MISR can also help assess the long-term stability of MODIS using Earth-scene targets. As discussed earlier, the primary advantage of using MISR is that the variations due to Earth-scene features (spatial and temporal) are significantly mitigated thereby decoupling the sensor behavior from any possible changes associated with the ground target.

The MODIS/MISR reflectance ratio for the spectrally matched bands is tracked over the mission lifetime using the ground targets discussed earlier. This approach essentially eliminates the impact of scene-dependence in tracking the consistency of the reflectance ratios. Figure 8 (a) and (b) show the MODIS/MISR reflectance ratio plotted as a function of time. The results for the four bands have been slightly offset on the time-scale for better clarity. A calendar year average has been performed on the derived ratios. Seasonal oscillations up to 3\% (peak-to-peak) are observed in the reflectance ratios in Figure 8 (a) before performing a yearly average. These oscillations are likely caused by the difference in the surface and atmospheric BRDF on the MODIS and MISR band pair. Since the goal is to evaluate the long-term stability of MODIS RSB, the multi-year seasonal oscillations can be tolerated. In the case of ocean scenes, the total radiance observed by the satellite is composed of 5-10 \% of ocean signal and 90-95\% of atmospheric signal. A larger spread in the MODIS/MISR ratios is observed for the ocean bands with band 16 showing the seasonal oscillations greater than $10 \%$. MODIS band 16 is primarily used in ocean color products to 
retrieve the aerosol optical thickness at $869 \mathrm{~nm}$. The water-leaving radiance in MODIS band 16 is negligible and the signal obtained is due to aerosols. The atmospheric and ocean surface scattering effects must be accurately modeled and removed to retrieve the net water-leaving radiance. Although multi-year continuous measurements are used in the analysis, the uncertainties caused due to these effects can bear a significant impact on the ocean band results.

An upward drift of about 3\% (2000-2015) is observed for the reflectance ratios of MODIS bands 1 and 2 with MISR.A similar drift of a lower magnitude ( 1.8\%) is observed in the case of MODIS bands 13 and 14. The longterm changes in the reflectance ratios associated with MODIS bands 4, 9, and 12 are observed to be within 2\%. Also, short-term deviations in these ratios are observed in the case of MODIS bands 4 and 9 in 2014 and band 1 in the early months of 2015.Although the assumption is that the MISR calibration is relatively stable over the mission lifetime, a more careful investigation is warranted to confirm this.

In order to decouple any impact due to a reflectance drift in MISR, MODIS TOA reflectance devoid of any MISR contribution is analyzed. The results do not exhibit any measurable drifts ( $>2 \%)$ for MODIS bands 1 and 2, therefore confirming that a drift in MISR reflectance resulted in the long-term drift of the ratio trends. MISR performed an assessment of its long-term radiometric stability using observations from the Egypt 1 desert site (27.12N, 26.10E). Results indicated a change per decade of $0.8 \%, 1.49 \%, 1.54 \%$ and $1.74 \%$ for the blue, green, red and NIR bands respectively. The total mission change in the radiance for the blue, green, red, and NIR bands was observed to be $1 \%,-1.9 \%, 2 \%$ and $2.3 \%$ respectively. However, the short-term deviation in MODIS band 1 (year 2015) is present in the reflectance trending of MODIS. MODIS band 1 uses Earth-view response trends from the Libyan desert to derive its on-orbit gain on a routine basis. This short-term deviation in the reflectance is attributed to the fitting algorithm update in the forward processing stream and is expected to be corrected in the next reprocessed version. As expected, the TOA reflectance trend from ocean sites yield a reflectance $<10 \%$ with prominent seasonal oscillations.

In order to quantify the presence of a long-term drift in the reflectance trends, regression lines were fitted and a statistical analysis of the slope of the regression line was performed. The slope of the regression line is tested for a null slope, which states that no long-term drift is observed, and alternative hypothesis, which states that the slope is significant and therefore indicates a temporal drift. The p-value denoting the probability of the test statistic is set at a $5 \%$ level under the following conditions:

(a) If the p-value $<0.05$, the null hypothesis ( $\mathrm{H} 0$ ) is rejected; therefore indicating a long-term drift in the reflectance trend

(b) If the p-value $\geq 0.05$, then it fails to reject the null hypothesis (H0); therefore indicating no long-term drift in the TOA reflectance trend.

Table II presents the result of the hypothesis testing confirming the absence of a measurable long-term drift in these MODIS bands. In all cases, the p-value is observed to be greater than 0.05 therefore failing to reject the null hypothesis. Also, included in the table is the long-term reflectance drift in \%. Performing a linear modeling fit to the data presented in Figure 8 and computing the difference in the modeled values between the first and the last year 
provides the long-term drift estimate. With the exception of bands 1 and 2, the total mission drift for other bands is within $2 \%$.

\section{Future Improvements}

Among the various techniques used to monitor the on-orbit calibration of satellite sensors, comparing nearsimultaneous scene pairs over stable ground targets with another sensor, described in this paper, is an effective approach to enhance the on-orbit calibration. Unlike previous efforts of comparing absolute differences between the two sensors, this work is more focused towards the use of MISR to benefit the on-orbit MODIS calibration for the RSB. By choosing simultaneous overpasses at the same view geometry over pseudo-invariant ground targets, most uncertainties associated with the day-to-day observations, such as variations in the atmospheric conditions or the impacts associated with the site's BRDF, are largely eliminated. As MODIS continues to operate far beyond its life expectancy, maintaining the accuracy of the on-orbit RSB calibration poses several challenges. One of the primary challenges is to continue characterizing the on-orbit RVS accurately. The MISR-based evaluation of the MODIS RVS for the spectrally matching bands indicates the on-orbit RVS stability to be within 1\% for MODIS bands 1, 2, 4 and 9. Similar results, with greater uncertainty of up to $3 \%$, are observed for bands $12,13,14$ and 16 . A more robust technique, yielding lower uncertainties is required to evaluate the on-orbit RVS performance for these bands.

The on-orbit calibration of the MODIS RSB does not account for the changes in the instrument's polarization sensitivity. The impacts are primarily observed at short wavelengths as observed in the mirror side differences in MODIS band 9. Work performed by Kwiatkowska et.al, and Lyapustin et.al, has shown that it is essential to account of the instrument's polarization sensitivity in order to maintain an accurate on-orbit calibration [18], [19]. Efforts are underway to implement a polarization correction to the Earth-scene data before the on-orbit RVS is derived. Work is also performed to develop alternative approaches for on-orbit RVS characterization. Using the MISR-based evaluation approach, an improvement in the algorithm to characterize the detector differences for MODIS band 9 has been identified. The differences, greater than $1 \%$, observed at nadir are primarily caused by the lack of a reliable calibration source between the SD and lunar measurements on either side of nadir. An algorithm involving the detector differences from Earth-view data at multiple scan-angles is being developed to account for these differences observed at nadir.

Finally, the temporal stability of the reflectance trends derived from the MODIS RSB is critical to maintain the quality of the various downstream products. In order to decouple MISR's long-term trends from MODIS, an independent confirmation solely relying on the MODIS reflectance trends was performed. With the exception of bands 1 and 2, the long-term stability of the MODIS bands presented in the paper is within 2\%. Short-term variations in the recent year caused by the forward updates were identified in bands 1 and 2 . These are expected to be corrected for in the next entire mission reprocess. 


\section{Summary and Conclusions}

The on-orbit calibration of the Terra MODIS RSB is tracked using regular measurements of its on-board calibrators supplemented by Earth-view measurements. The measurements from the on-board calibrators primarily rely on the SD and lunar measurements and the Earth-view response trends at multiple AOI are derived from pseudo-invariant desert sites. Uncertainties associated with the use of Earth-view targets for on-orbit calibration are well documented and need a constant and careful tracking. MISR, also on the Terra spacecraft, provides an effective means of tracking the MODIS on-orbit calibration. This paper demonstrates the use of MISR as an additional on-board calibrator to monitor the on-orbit calibration consistency of MODIS RSB. Due to its push-broom mechanism, simultaneous MISR collects effectively eliminate the impacts of scene dependence. The North Atlantic ocean and North African desert sites are selected to evaluate the on-orbit performance of the MODIS RVS characterization. Results indicate that the MODIS/MISR ratio between the two ends of the MISR swath demonstrates a stable behavior over time with a temporal change within $1 \%$ for all bands. A higher standard deviation is observed for the bands using ocean sites due to atmospheric effects. The reflectance differences between the two sides of the MODIS scan mirror exhibit changes $<1 \%$ for all bands with the exception of MODIS band 9. The changing polarization sensitivity of the instrument impacts the mirror side differences observed for band 9. An improvement to the current on-orbit RVS characterization methodology has been identified to ensure a more accurate characterization of the detector-differences at nadir. With the exception of band 9, the detector differences for the RSB reflectance presented here are observed to be within 1\%. Finally, the temporal stability of MODIS RSB is assessed using MODIS/MISR reflectance ratios to decouple the impact of ground target variations. The ratios exhibited a 3\% drift in the case of MODIS bands 1 and 2 and less than 2\% drift for the other bands. To confirm these results, MODISreflectance trending without the MISR normalization has been monitored for long-term consistency. A hypothesis testing is performed to confirm that no measurable long-term drift is observed for all of the RSB presented here. 


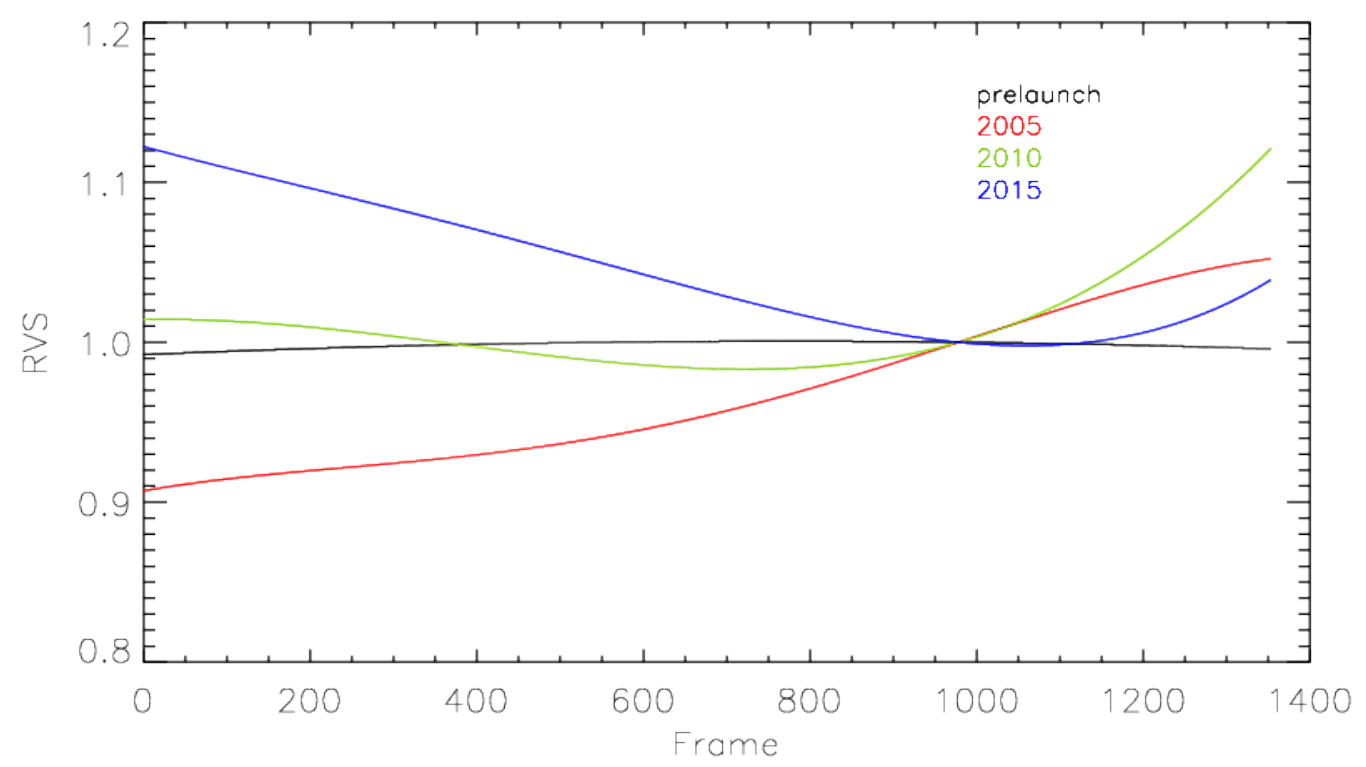

Figure 1a. Prelaunch and on-orbit RVS for Terra band 9

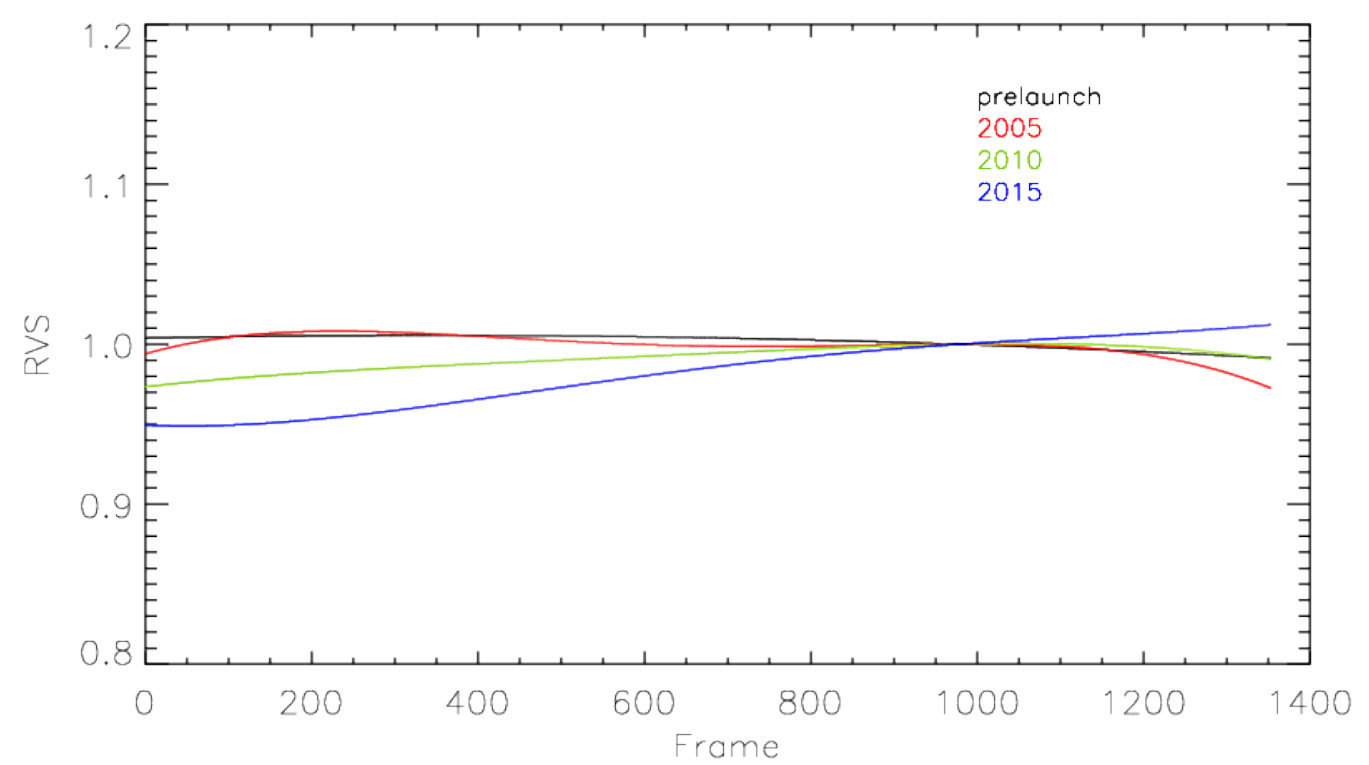

Figure 1b. Prelaunch and on-orbit RVS for Terra band 4 


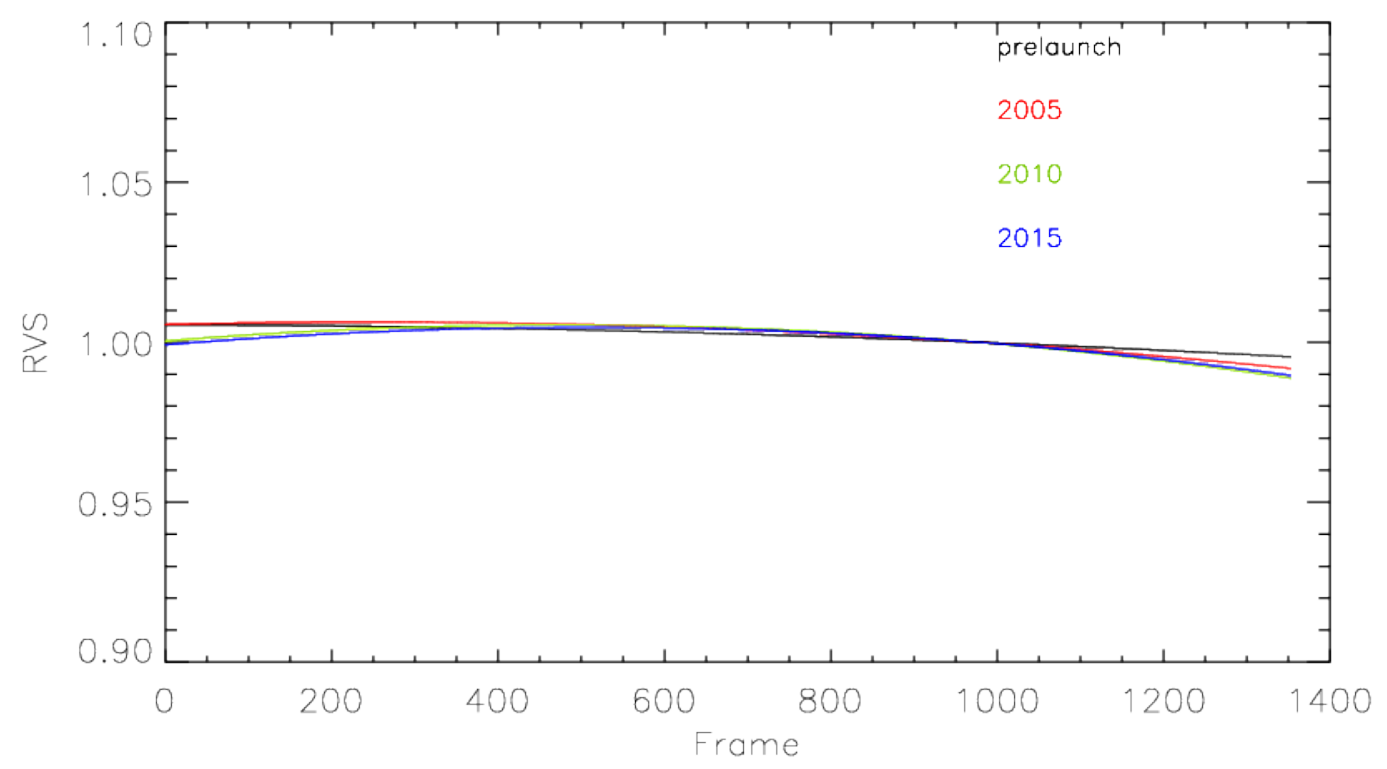

Figure 1c. Prelaunch and on-orbit RVS for Terra band 1

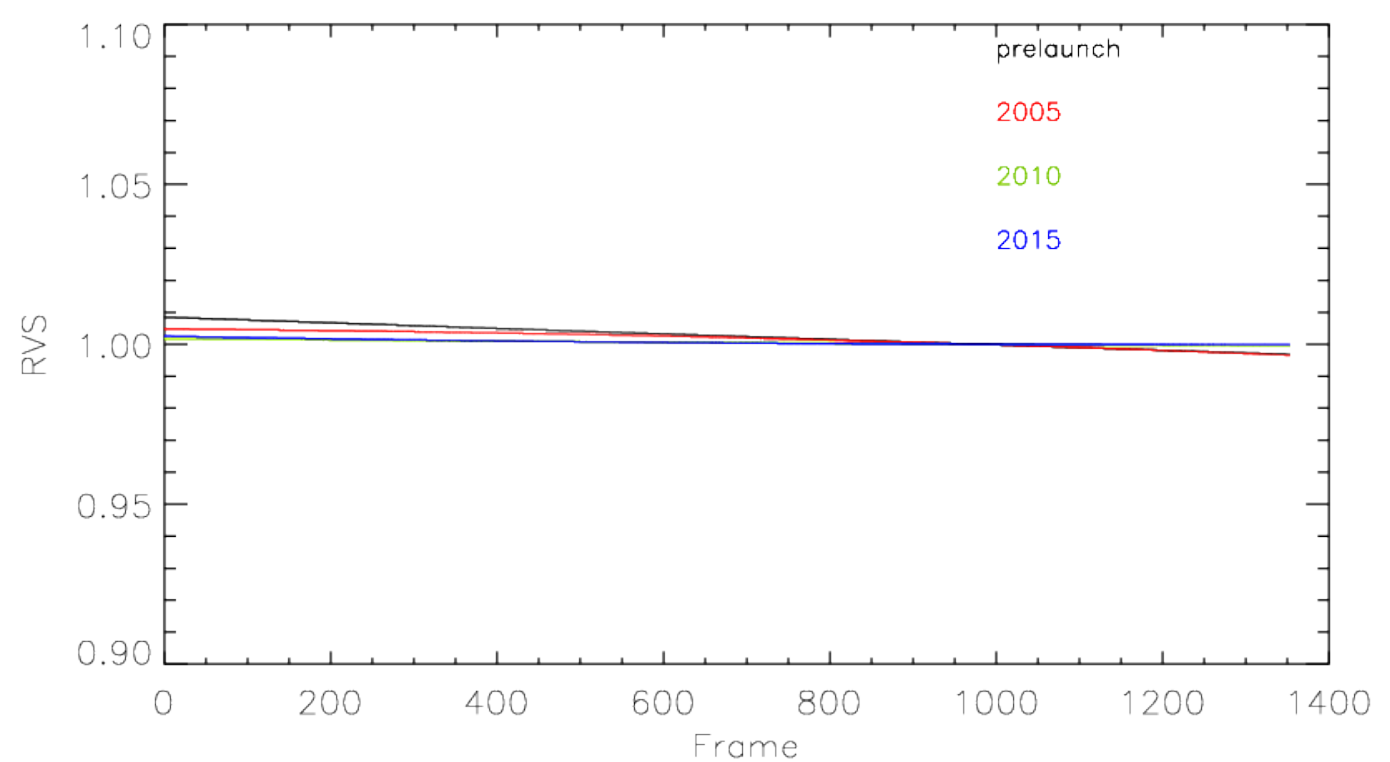

Figure 1d. Prelaunch and on-orbit RVS for Terra band 2 

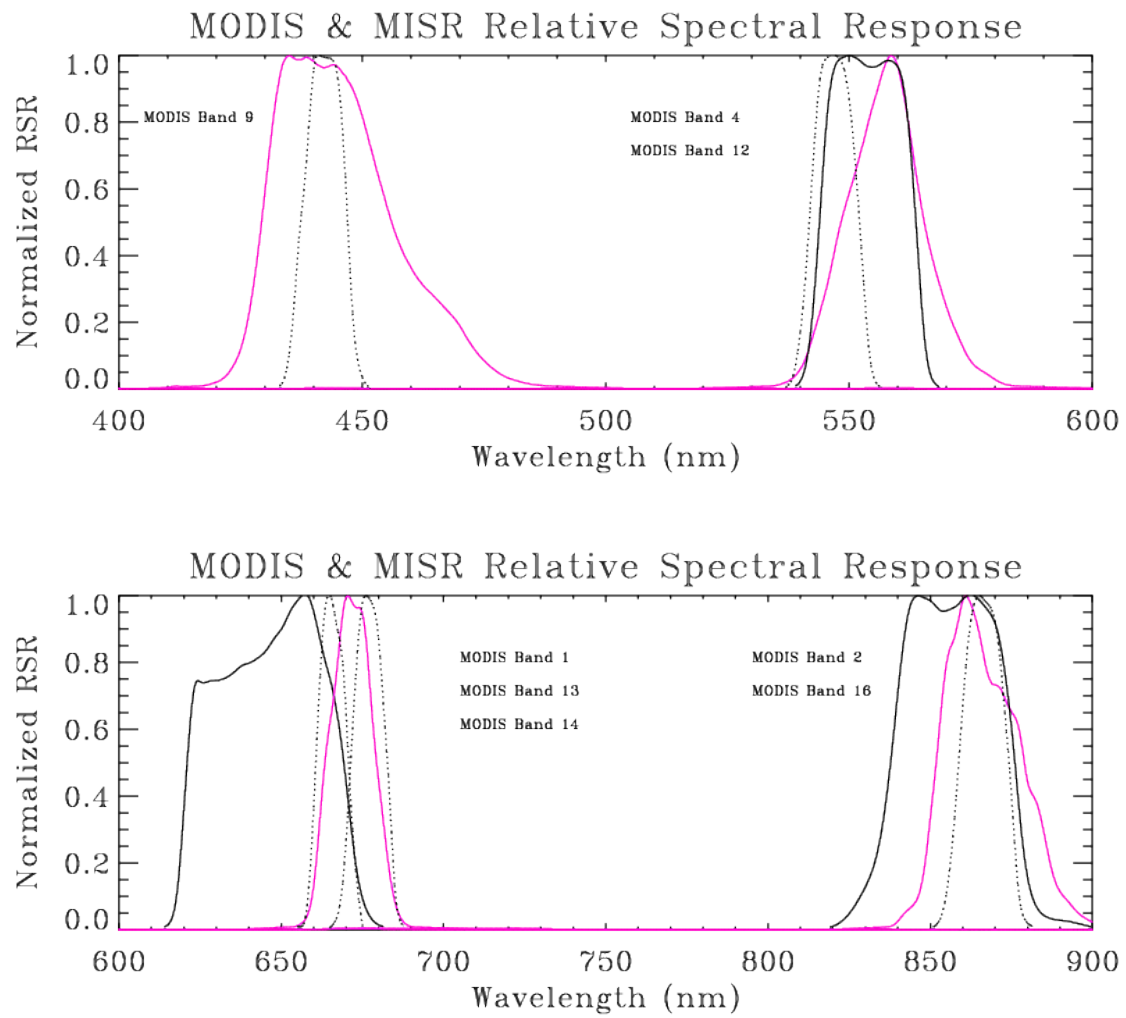

Figure 2 MODIS-MISR Relative Spectral Response comparison

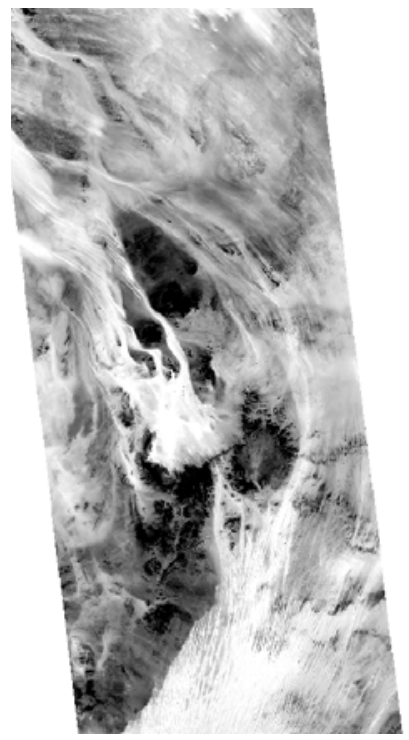

Figure 3a. North African desert overpass from day 186 of the year 2015

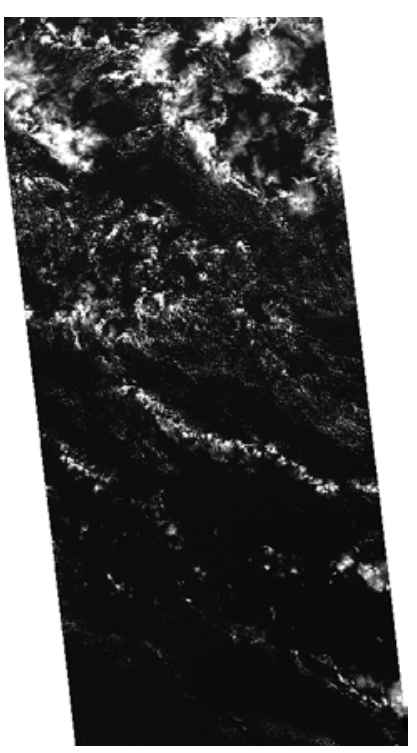

Figure 3b. North Atlantic ocean overpass from day 20 of the year 2005 


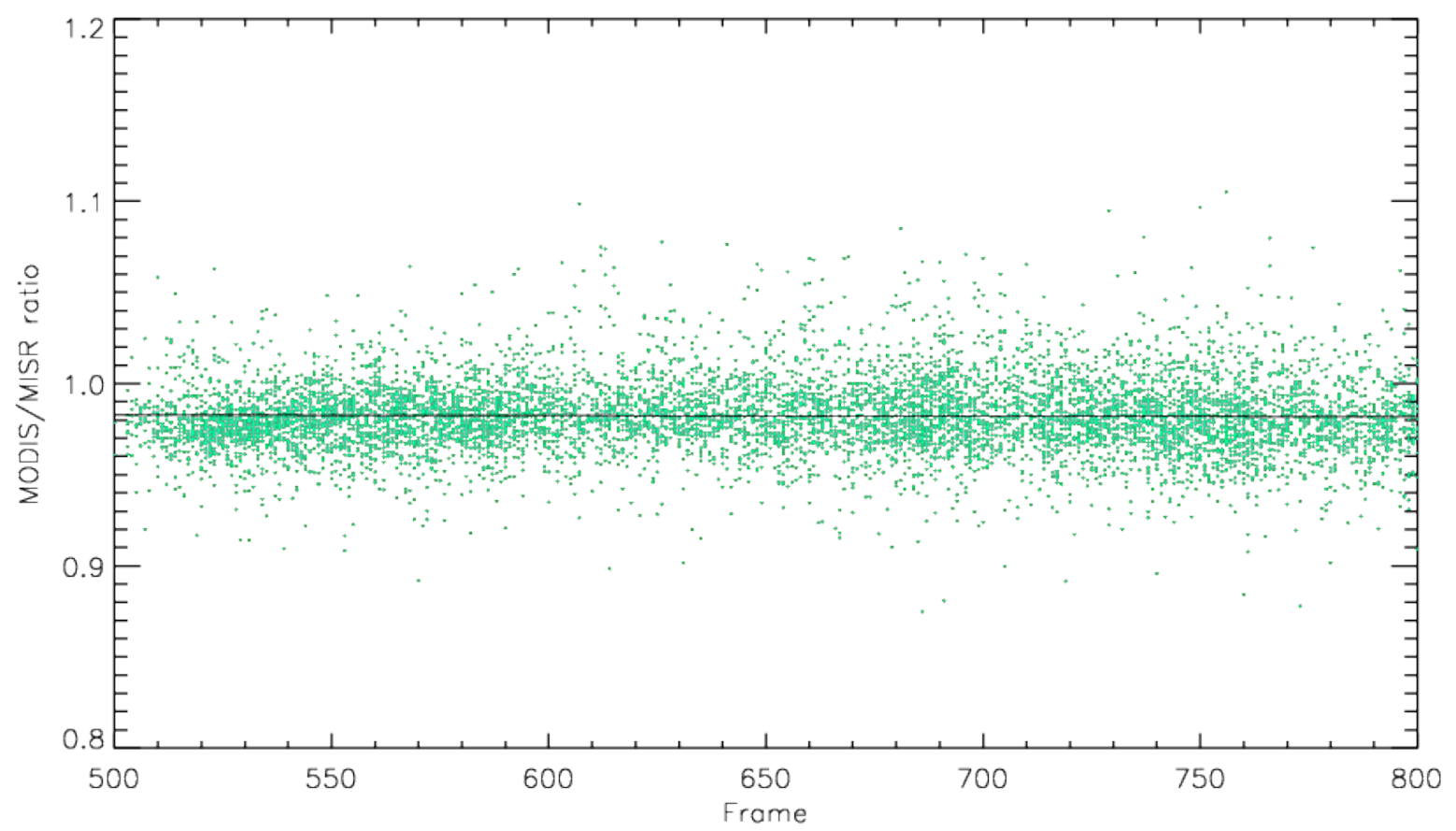

Figure 4a . MODIS/MISR ratio for band 9 over a simultaneous desert overpass from 2005 as a function of MODIS frames number (scan-angle). All the matched pixels from mirror side 1 are plotted along with a linear regression.

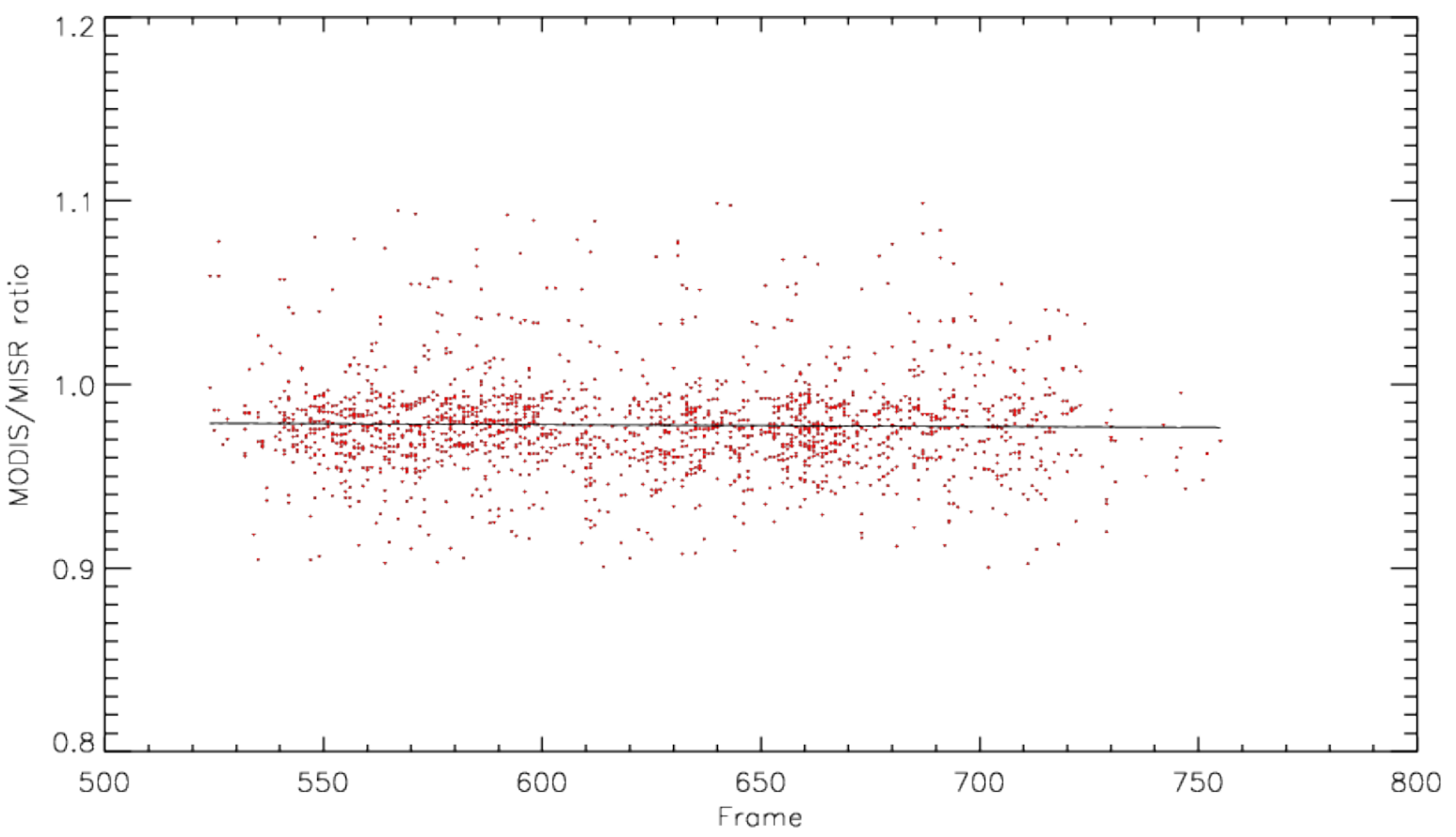

Figure 4b . MODIS/MISR ratio for band 13 over a simultaneous desert overpass from 2005 as a function of MODIS frames number (scan-angle). All the matched pixels from mirror side 1 are plotted along with a linear regression. 

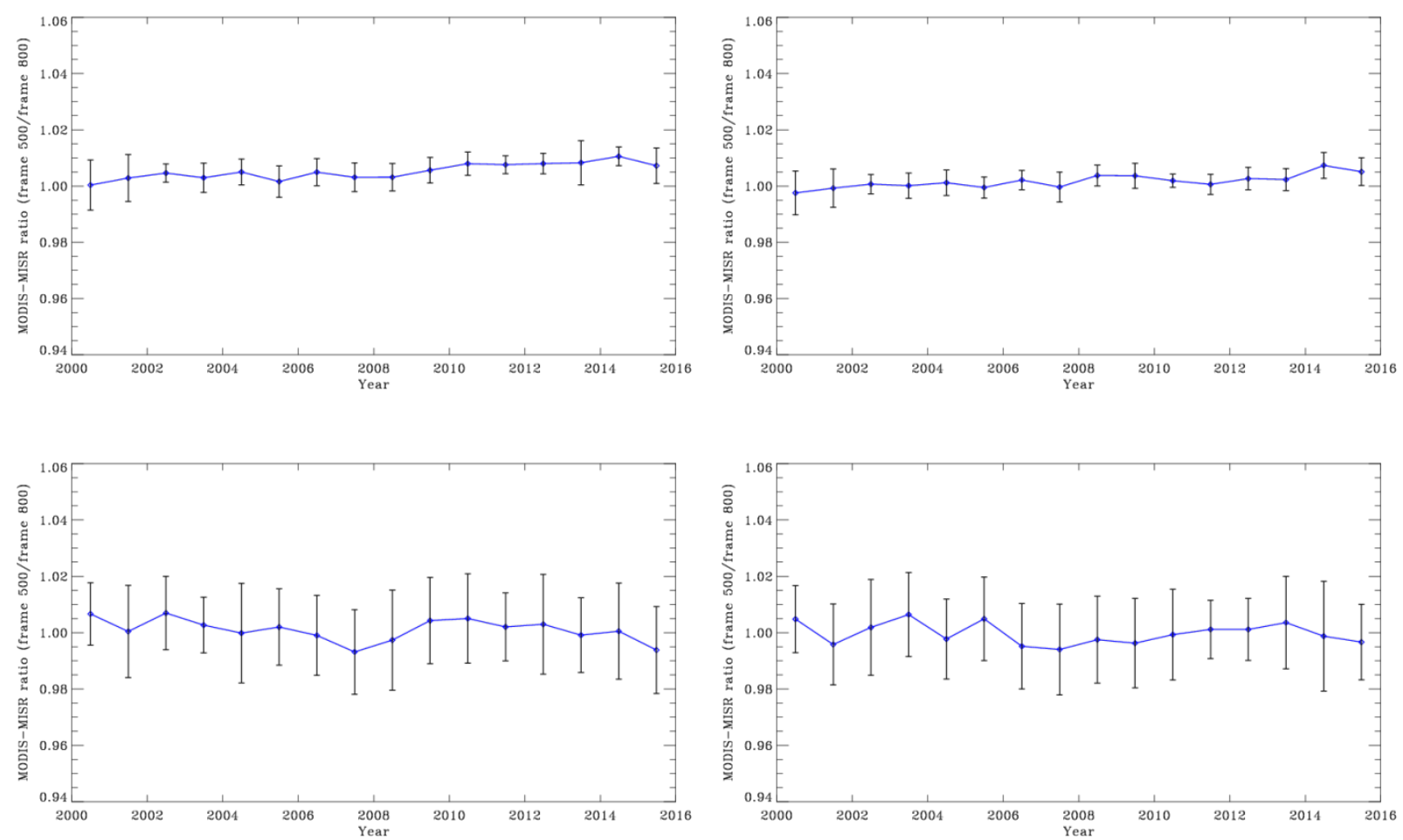

Figure5. (a) Relative difference between the MODIS/MISR ratio at frame 500 and frame 800. A calendar-year average relative difference is plotted for mirror side 1 of band 1 for all North African desert scene-pairs. (b) Results for MODIS band 4 over the North African desert. (c) Results for MODIS band 13 over the North Atlantic ocean are plotted. (d) Results for MODIS band 14 over North Atlantic ocean. 


\begin{tabular}{|c|c|c|c|c|c|c|c|c|c|}
\hline & & \multicolumn{2}{|c|}{2000} & \multicolumn{2}{c|}{2005} & \multicolumn{2}{c|}{2010} & \multicolumn{2}{|c|}{2015} \\
\hline Band & MS & Rel. Diff & Std.dev & Rel. Diff & Std.dev & Rel. Diff & Std.dev & Rel. Diff & Std.dev \\
\hline B1 & 1 & 1.003 & 0.008 & 1.002 & 0.006 & 1.008 & 0.004 & 1.007 & 0.006 \\
\hline B1 & 2 & 1.004 & 0.006 & 1.003 & 0.005 & 1.007 & 0.004 & 1.007 & 0.005 \\
\hline B2 & 1 & 1.003 & 0.007 & 1.003 & 0.008 & 1.012 & 0.005 & 1.011 & 0.007 \\
\hline B2 & 2 & 1.004 & 0.006 & 1.003 & 0.004 & 1.012 & 0.006 & 1.012 & 0.006 \\
\hline B4 & 1 & 0.999 & 0.007 & 0.999 & 0.004 & 1.002 & 0.002 & 1.005 & 0.005 \\
\hline B4 & 2 & 0.999 & 0.006 & 1.001 & 0.006 & 1.001 & 0.003 & 1.006 & 0.005 \\
\hline B9 & 1 & 0.999 & 0.006 & 0.999 & 0.004 & 0.996 & 0.005 & 0.997 & 0.006 \\
\hline B9 & 2 & 0.998 & 0.005 & 0.999 & 0.003 & 1.000 & 0.004 & 0.997 & 0.005 \\
\hline B12 & 1 & 1.007 & 0.013 & 1.006 & 0.013 & 1.006 & 0.013 & 1.010 & 0.015 \\
\hline B12 & 2 & 1.005 & 0.016 & 1.005 & 0.017 & 0.995 & 0.012 & 1.012 & 0.014 \\
\hline B13 & 1 & 1.000 & 0.016 & 1.002 & 0.014 & 1.005 & 0.016 & 0.994 & 0.015 \\
\hline B13 & 2 & 0.997 & 0.017 & 0.991 & 0.011 & 0.998 & 0.015 & 1.002 & 0.013 \\
\hline B14 & 1 & 0.996 & 0.014 & 1.005 & 0.015 & 0.999 & 0.016 & 0.997 & 0.013 \\
\hline B14 & 2 & 0.997 & 0.015 & 1.003 & 0.016 & 1.004 & 0.019 & 0.993 & 0.010 \\
\hline B16 & 1 & 0.998 & 0.013 & 1.008 & 0.013 & 0.987 & 0.018 & 1.008 & 0.017 \\
\hline B16 & 2 & 0.988 & 0.012 & 1.014 & 0.006 & 1.005 & 0.019 & 0.990 & 0.015 \\
\hline
\end{tabular}

Table I. Summary of the MODIS RSB RVS assessment using MISR. The results for bands 1, 2,4 and 9 were derived using the desert site and the ocean site was used for bands 12,13, 14 and 16. Results from four representative years are presented (yearly-average over the calendar year). The standard deviation denotes the variation around the mean value of relative difference within the year. 

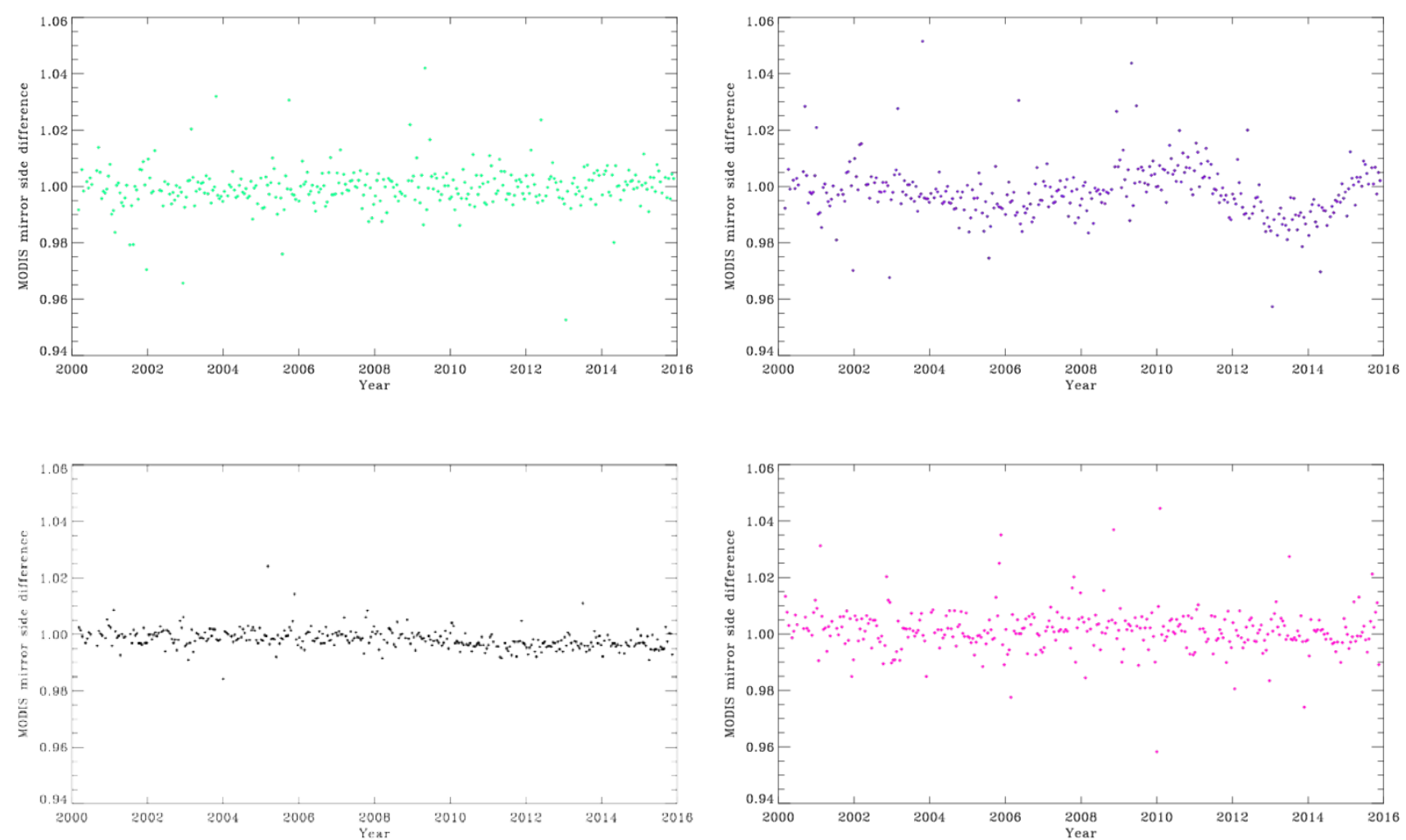

Figure6 (a) MODIS mirror side differences for band 4, (b) band 9, (c) band 13, and (d) band 16.
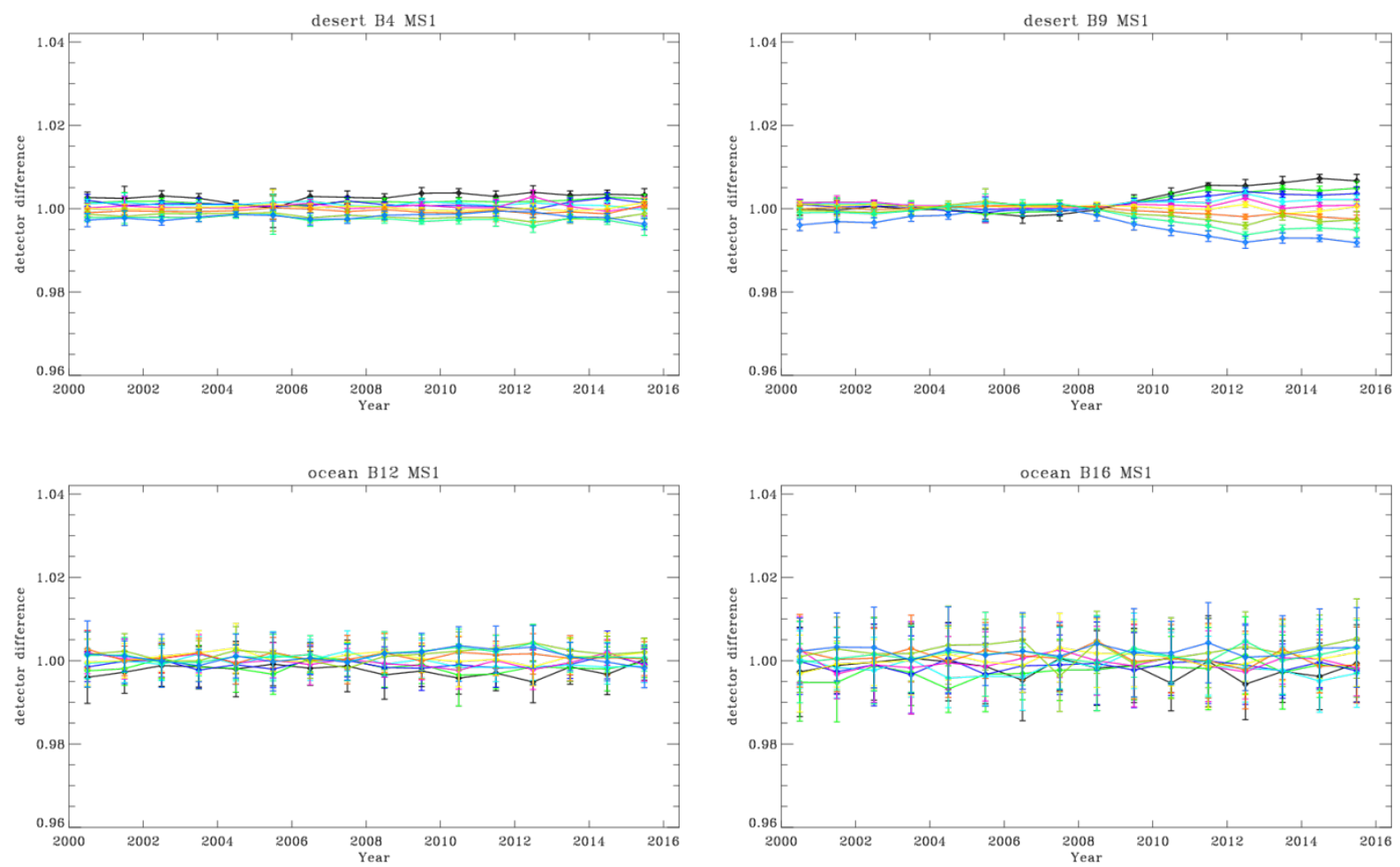

Figure 7 (a) MODIS detector-detector differences for band 4, (b) band 9, (c) band 13, and (d) band 16. 

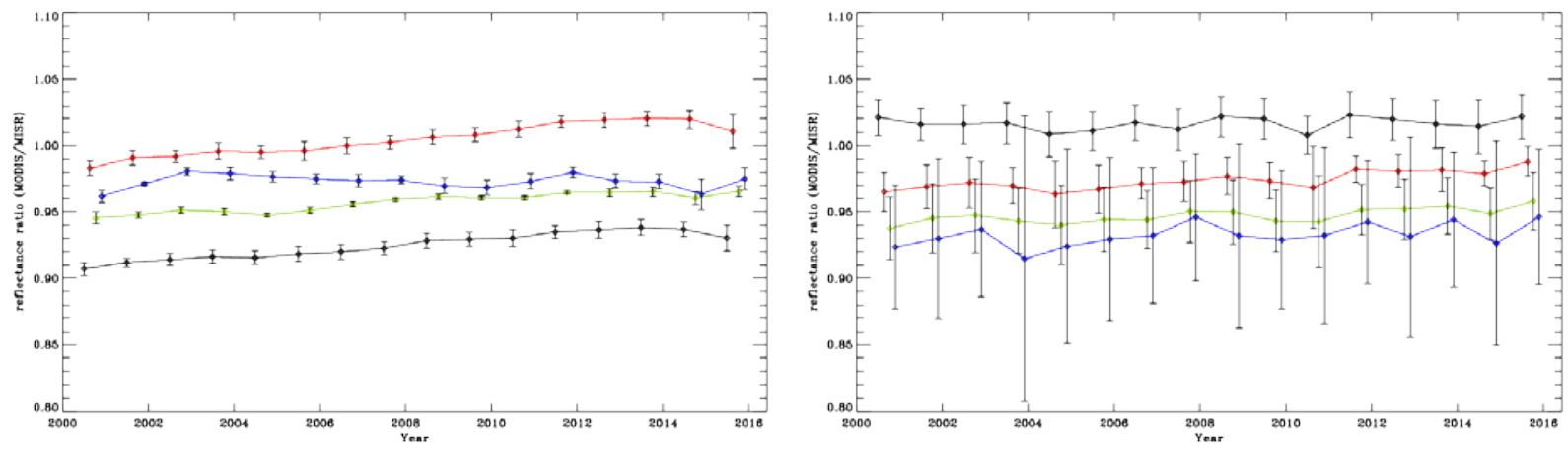

Figure 8(a) MODIS/MISR reflectance ratio trending for bands 1 (black),2 (red),4 (green), and 9 (blue) over North African desert (b) MODIS/MISR reflectance ratio trending for bands 12 (black),13 (red),14 (green), and 16 (blue) over North Atlantic ocean.

\begin{tabular}{|c|c|c|c|c|}
\hline MODIS Band & t-value & p-value & H0, slope = 0 & Total Mission Drift (\%) \\
\hline Band 1 & 2.939 & 0.998 & Fail to Reject & $3.27 \%$ \\
\hline Band 2 & 3.207 & 0.999 & Fail to Reject & $3.52 \%$ \\
\hline Band 4 & 1.475 & 0.929 & Fail to Reject & $1.78 \%$ \\
\hline Band 9 & -0.084 & 0.466 & Fail to Reject & $0.09 \%$ \\
\hline Band 12 & 1.46 & 0.927 & Fail to Reject & $0.25 \%$ \\
\hline Band 13 & -1.609 & 0.054 & Fail to Reject & $1.89 \%$ \\
\hline Band 14 & 0.509 & 0.695 & Fail to Reject & $1.35 \%$ \\
\hline Band 16 & -1.464 & 0.072 & Fail to Reject & $1.52 \%$ \\
\hline
\end{tabular}

Table II: Hypothesis testing for the long-term reflectance trends. Also, included is the total mission drift (\%) from 2000-2015.

\section{Acknowledgements}

The MISR datasets were obtained from the NASA Langley Research Center Atmospheric Science Data Center. The authors would also like to thank Dr. Jeff McIntire, SSAI for technical review of this manuscript.

\section{References}

[1] Xiong, X., and Barnes, W.L., An Overview of MODIS radiometric calibration and characterization, Advances in Atmospheric Sciences, 23(1), 69-79, (2006).

[2] Xiong, X., M. D. King, V.Salomonson, W. Barnes, B. N. Wenny, A. Angal, A. Wu, S. Madhavan, and D. Link, "Moderate Resolution Imaging Spectroradiometer on Terra and Aqua Missions", John Wiley \& Sons, Ltd, vol. 9781118945179, pp. 53-89, 2015.

[3] Bruegge, C. J., Abdou, W. A., Diner, D. J., Gaitley, B. J., Helmlinger,M. C., Kahn, R. A., and Martonchik, J. V.: Validating theMISR radiometric scale for the ocean aerosol science communities,in: Post-Launch Calibration of Satellite Sensors, edited by:Morain, S. A. and Budge, A. M., A. A. Balkema Publishers, Leiden,the Netherlands, 103-115, 2004. 
[4] Bruegge, C. J., Diner, D. J., Kahn, R. A., Chrien, N., Helmlinger,M. C., Gaitley, B. J., and Abdou, W. A.: The MISR radiometriccalibration process, Remote Sens. Environ., 107, 2-11,doi:10.1016/j.rse.2006.07.024, 2007.

[5] Diner, D. J., Kahn, R. A., Bruegge, C. J., Martonchik, J. V.,Abdou, W. A., Gaitley, B. J., Helmlinger, M. C., Kalashnikova,O. V., and Li, W.-H.: Refinements to MISR's radiometriccalibration and implications for establishing a climate-qualityaerosol observing system, SPIE Proc., 5652, 57-65, 2004.

[6] Bruegge, C. J., Diner, D. J., Gray, E., Jovanovic, V., Gray, E., Di Girolamo, L., and Zhao, G.: Radiometric stability of the Multi-angle Imaging SpectroRadiometer (MISR) following 15 years on-orbit, Proc. SPIE 9218, Earth Observing Systems XIX, 92180N, doi:10.1117/12.2062319, 2014.

[7] Wu, A., A. Angal, and X. Xiong, "Comparison of coincident MODIS and MISR reflectances over the 15-year period of EOS Terra", Proc. SPIE 9218, Earth Observing Systems XIX, 92180W, 2014.

[8] Xiong, X., B. N. Wenny, A. Wu, A. Angal, X. Geng, H. Chen, J. Dodd, D. Link, S. Madhavan, N. Chen, et al., "Status of Terra MODIS operation, calibration, and performance ", Proc. SPIE 9218, Earth Observing Systems XIX, 92180O, 2014.

[9] Czapla-Myers, J. S,N. P. Leisso,N J. Anderson, SF. Biggar, "On-orbit radiometric calibration of Earth-observing sensors using the Radiometric Calibration Test Site (RadCaTS)”, Proc. SPIE 8390, Algorithms and Technologies for Multispectral, Hyperspectral, and Ultraspectral Imagery XVIII, 83902B (May 8, 2012), doi:10.1117/12.918614.

[10] Toller, G., X. Xiong, J. Sun, B. N. Wenny, X. Geng, J. Kuyper, A. Angal, H. Chen, S. Madhavan, and A. Wu, "Terra and Aqua Moderate-resolution Imaging Spectroradiometer Collection 6 Level 1B Algorithm", Journal of Applied Remote Sensing, vol. 7, issue 1, 2013.

[11] Sun, J., X. Xiong, W. Barnes, and B. Guenther, "MODIS Reflective Solar Bands On-Orbit Lunar Calibration", IEEE Trans. Geosci. Remote Sens., vol. 45, issue 7, pp. 2383-2393, 2007.

[12] Sun, J., X. Xiong, A. Angal, H. Chen, A. Wu, and X. Geng, "Time-Dependent Response Versus Scan Angle for MODIS Reflective Solar Bands", IEEE Transactions on Geoscience and Remote Sensing, vol. 52, issue 6, pp. 31593174, 2014.

[13] Xiong, X., J. Sun, W. Barnes, V. Salomonson, J. Esposito, H. Erives, and B. Guenther, "Multiyear On-Orbit Calibration and Performance of Terra MODIS Reflective Solar Bands", IEEE Trans. Geosci. Remote Sens., vol. 45, issue 4, pp. 879-889, 2007.

[14] Geng, X., A. Angal, J. Sun, A. Wu, T. Choi, and X. Xiong, "Characterization of MODIS mirror side difference in the reflective solar spectral region", Proceedings of SPIE - Earth Observing Systems XVI, vol. 8153, no. 81531O, 2011.

[15] Wu, A., X. Xiong, A. Angal, and W. Barnes, "Evaluation of Detector-to-Detector and Mirror Side Differences for Terra MODIS Reflective Solar Bands Using Simultaneous MISR Observations", Int. J. Remote Sens., vol. 32, issue 2, pp. 299-312, 2011.

[16] Sun, J., and X. Xiong, "MODIS Polarization Sensitivity Analysis", IEEE Trans. Geosci. Remote Sens., vol. 45, no. 9, pp. 2875-2885, 2007. 
[17] Angal, A., J. Sun, X. Geng, M. Chu, and X. Xiong, "Assessment of the MODIS RSB detector differences using earth-view targets ", Proc. SPIE 8866, Earth Observing Systems XVIII, 88661T, 2013.

[18] Kwiatkowska, E., B. A. Franz, G. Meister, C. McClain, and X. Xiong, "Cross Calibration of Ocean-color Bands from Moderate Resolution Imaging Spectroradiometer on Terra Platform", Appl. Opt, vol. 47, pp. 6796-6810, 2008.

[19] Lyapustin, A., Y. Wang, X. Xiong, G. Meister, S. Platnick, R. Levy, B. A. Franz, S. Korkin, T. Hilker, J. Tucker, et al., "Scientific impact of MODIS C5 calibration degradation and C6 improvements", Atmospheric Measurement Techniques Discussions, vol. 7, issue 7, pp. pp.7281-7319, 2014.

[20] Wu, A., X. Geng, A.E.Wald, and X. Xiong, "Tracking Terra MODIS on-orbit polarization sensitivity using pseudo-invariant desert sites ", Proc. SPIE 9639, Sensors, Systems, and Next-Generation Satellites XIX, 963914, 2015. 